\title{
Effect of Deposition Parameters on the Structure of ZnCo Alloy Nanowires and Growth Mechanism
}

\author{
Tahir Mehmood ${ }^{1}$, K. M. Wu ${ }^{1, \text { * , Aiman Mukhtar }}{ }^{1}$, Marina Afzal ${ }^{2}$, Babar Shahzad Khan ${ }^{2, ~ * ~}$ \\ ${ }^{1}$ The State Key Laboratory of Refractories and Metallurgy, Hubei Province Key Laboratory of Systems Science in Metallurgical Process, \\ International Research Institute for Steel Technology, Wuhan University of Science and Technology, Wuhan, P. R. China \\ ${ }^{2}$ Department of Physics, Government College Women University, Sialkot, Punjab, Pakistan
}

\section{Email address:}

tahir10621@yahoo.com, (T.Mehmood),wukaiming@wust.edu.cn(K.M. Wu), babar.shahzad.khan@gcwus.edu.pk(B.S. Khan)

${ }^{*}$ Corresponding author

\section{To cite this article:}

Tahir Mehmood, K. M. Wu, Aiman Mukhtar, Marina Afzal, Babar Shahzad Khan. Effect of Deposition Parameters on the Structure of ZnCo Alloy Nanowires and Growth Mechanism. Journal of Electrical and Electronic Engineering. Vol. 8, No. 1, 2020, pp. 10-15.

doi: $10.11648 /$ j.jeee.20200801.12

Received: December 13, 2019; Accepted: January 3, 2019; Published: January 4, 2019

\begin{abstract}
To elucidate the effect of voltage and concentration on the elctrodeposition of ZnCo nanowires, we have studied the deposition of ZnCo nanowires by XRD and SEM. The structure of ZnCo alloy nanowires changes by changing the concentration of electrolyte as $100-\mathrm{x} g / 1 \mathrm{Co}_{100-\mathrm{x}} \mathrm{Zn}_{\mathrm{x}}(0 \leq \mathrm{x} \leq 15)$, at constant voltage -1.6 , and fixed $\mathrm{pH} 3$. XRD results show that change in concentration can transform the crystal structure of electrodeposited $\mathrm{Co}_{100-\mathrm{x}} \mathrm{Zn}_{\mathrm{x}}$ from crystalline to amorphous. Furthermore, similar behavior from crystalline to amorphous was also observed for ZnCo electrodeposited nanowires at various potentials $(-1.6 \mathrm{~V},-2.5 \mathrm{~V}$, and $-3.0 \mathrm{~V})$ from the bath containing $\mathrm{Co}_{95 \mathrm{~g} / 1}$ and $\mathrm{Zn}_{5 \mathrm{~g} / \mathrm{l}}, \mathrm{pH} 3$ and at room temperature. We argued that this change in structure of $\mathrm{ZnCo}$ nanowires can be attributed to the faster growth rate of $\mathrm{Zn}$ than Co. These results can be explained by the electron tunneling theory. The workfunction of $\mathrm{Zn}(4.33 \mathrm{eV})$ is smaller than $\mathrm{Co}(5.0 \mathrm{eV})$ and the wavefunction of $\mathrm{Zn}$ metal has a larger overlap with orbital wavefunction of $\mathrm{Zn}$ ions, which leads the faster growth rate of $\mathrm{Zn}$ and hence influenced the structure of $\mathrm{ZnCo}$ alloy nanowires.
\end{abstract}

Keywords: Alloy Nanowires, Electrodeposition, Growth, Electron Tunneling

\section{Introduction}

Cobalt is naturally occurring magnetic metals at a higher temperature $1100^{\circ} \mathrm{c}$ than any other metals. while Zinc is used as basic element of electronic appliances due to its controlled conductivity at high temperature. Unique characteristics of $\mathrm{ZnCo}$ alloy made it valuable for various technologies. The 1$\mathrm{D}$ metallic alloy nanowires have gain much attention due to exceptional features which are different from other geometrical forms [46] having countless potential uses at industries, such as electronic appliances, ultra-high density memory devices [47] and sensors [48] etc. Conventional technologies based device, such as the p-n junction diodes [1], FETs [2], logic gates [1], and LEDs [3, 4] have been fabricate in small size using various nanomaterials such as ZnCo nanowires, illustrating their function as the building blocks headed for the production of complex integrated circuits applying the "bottom-up" pattern [4]. Metallic ZnCo alloy nanowires linked to electron field emission [5], such as the less diameter ratio and great curvature at the tip of nanowire, due to least threshold voltage for the electron emission [6], which demonstrate extreme field emission currents at narrow tip (almost 10nm radius) of a $\mathrm{Si}$ cone. The spectacular lattice of $\mathrm{ZnCo}$ alloy can be applicable in light reflecting diodes [7]. The large surface morphology and unique electrical nature of one dimension $\mathrm{ZnCo}$ alloy nanowires have appropriate inorganic-organic solar cell [8]. Solar cell manufactured using $\mathrm{ZnCo}$ alloy show unusual effectiveness [9]. Metallic $\mathrm{Zn}$ alloy prepared by using various metal parts like $\mathrm{Co}, \mathrm{Ni}, \mathrm{Pd}, \mathrm{Ag}, \mathrm{Cu}, \mathrm{Au}$ etc. applicable such as sensors [10] for different optical examine outs. The improved thermo power and multifunctional act in the $\mathrm{ZnCo}$ alloy nanowires made them most striking for thermoelectric energy alteration devices and cooling system [11]. Various types of single domain magnetic $\mathrm{ZnCo}$ alloy 
nanowires can manufactured with controlled (length and diameter) morphology related with common dimensions and well-sequenced in a close-packed array can be measured to gain countless magnetic uses [12, 13].

A lot of studies show the fabrication of $\mathrm{ZnCo}$ alloy nanowires. Zhu et al, fabricate the $\mathrm{ZnCo}$ alloy nanowires using Dc electrodeposition technique and studied the influence of applied voltage on the morphology and crystal structure of deposited $\mathrm{ZnCo}$ alloy nanowires. Their results reveal that the increasing applied voltage change the amorphous $\mathrm{ZnCo}$ alloy nanowires into hcp crystalline phase [14]. Koohbor et al examined the co-electrodeposition of highly arranged $\mathrm{Co}_{1-\mathrm{x}} \mathrm{Zn}_{\mathrm{x}}$ nanowires into porous layer of anodized aluminum oxide templates. They studied consequence of $\mathrm{Zn}$ content on the crystal structure and magnetic properties. Their results illustrate that rise in the concentration of $\mathrm{Zn}$ content of electrolyte forces the hcp crystal structure of Co nanowires into the amorphous phase of $\mathrm{ZnCo}$ electrolyte, displaying significant reduction in magnetic properties just like low Hc value (high coercivity) [15]. Heydari et al studied the effect of pulse current parameters on $\mathrm{ZnCo}$ alloy coating (current-off time, current-on time, and peak current density on the structural morphology and phase conversion [16]. Xua [2010] fabricate the amorphous $\mathrm{Co}-\mathrm{Zn}$ alloy nanowires in AAO templates Their results indicate when the applied magnetic field is along the wire axis, the squareness and coercivity of the nanowire arrays reduced with increasing $\mathrm{Zn}$ ion concentration [17]. Karahan et al investigated the change in magnetic properties, microstructure and phase contents of $\mathrm{Zn}$-Co electrodeposition. Various concentration of cobalt content in the electrolytic solution can improve the corrosion resistance due to its uni-axial crystal phase and its better structural analysis [18]. Almasi and his co-worker explained magnetic function of co-deposited and annealed $\mathrm{Co}_{x} \mathrm{Zn}_{1-x}$ alloy nanowires using first-order reversal curve (FORC) technique. It was observed that, by increasing $\mathrm{Zn}$ content the magnetostatics link and coercivity distribution, squareness average coercivity, shifted to low values. [19]. Kashi et al fabricated CoZn alloy nanowires embedded in anodic aluminum oxide template using AC pulse electrodeposition and studied effect of pulse off time in electrolyte with fixed concentration of $\mathrm{Co}^{+2}$ and $\mathrm{Zn}^{+2}$ at same $\mathrm{pH} 4$ and show that higher deposition of Co content is gained by increasing off time pulses [20]. Xu et al fabricate the $\mathrm{Co}_{1-\mathrm{x}} \mathrm{Zn}_{\mathrm{x}}$ nanowire in $\mathrm{AAO}$ template by electrodeposition and indicated that the structural morphology of nanowires is amorphous.

The structure of $\mathrm{ZnCo}$ electrodeposited nanowires changes from crystalline to amorphous, however, the reason for this structural change is not well understood. We have proposed in the previous paper that the growth of metal nanowires can be related to the dehydration of hydrated metal ions which involves valence electrons tunneling to hydrated metal ions $[21,22]$, leading to neutralization of the hydrated metal ions. The neutral metal atoms are adsorbed on the surface and then diffuse to surface sites (such as kink site) where they incorporate into the metal lattice, thus leading to the growth [22]. Since the probability of electron tunneling is related to the workfunction of metals, the workfunction can have a significant effect on the growth of metal alloy nanowires. Moreover, the elemental processes of electrodeposition are still not well understood. Therefore, the present paper attempts to study the workfunction effect of metal alloy nanowire growth and to understand the electrode reaction mechanism.

\section{Experimental Details}

The detailed experimental procedure for preparation of anodic alumina oxide (AAO) templates has been given in our previous papers [23-25]. The templates obtained by the above method have cylindrical and hexagonally arranged pores of about $50 \mathrm{~nm}$ in diameter and the length of the pore is about $65 \mu \mathrm{m}$. In order to deposit metal into the pores of AAO templates, $\mathrm{Au}$ film was sputtered onto the back side of the AAO templates to serve as a working electrode.

To study the growth of $\mathrm{ZnCo}$ nanowires, the effect of concentration and potential was varied. The electrolyte for deposition of $\mathrm{ZnCo}$ nanowires with various concentration $\left(100-\mathrm{x} \mathrm{g} / \mathrm{l}: \mathrm{Co}_{100-\mathrm{x}} \mathrm{Zn}_{\mathrm{x}}\right)$ was prepared by using $100 \mathrm{~g} / \mathrm{l}$ $\mathrm{CoSO}_{4} \cdot 7 \mathrm{H}_{2} \mathrm{O}+\mathrm{x}-\mathrm{ZnSO}_{4} \cdot 7 \mathrm{H}_{2} \mathrm{O}$ and $45 \mathrm{~g} / 1 \mathrm{H}_{3} \mathrm{BO}_{3}$. The potentiostatic deposition was conducted in a two-electrode cell at $-1.6 \mathrm{~V}$. To study the potential effect $\mathrm{ZnCo}$ alloy nanowires were deposited at various voltages $(-1.6 \mathrm{~V},-2.0 \mathrm{~V}$ and $-3.0 \mathrm{~V})$. The electrolyte for deposition $95 \mathrm{~g} / 1 \mathrm{CoSO}_{4} \cdot 7 \mathrm{H}_{2} \mathrm{O}$ $+5 \mathrm{~g} / \mathrm{ZnSO}_{4} .7 \mathrm{H}_{2} \mathrm{O}$ and $45 \mathrm{~g} / 1 \mathrm{H} 3 \mathrm{BO}_{3}$. The $\mathrm{pH}$ of electrolytes was adjusted to 3 by adding $1 \mathrm{M} \mathrm{H}_{2} \mathrm{SO}_{4}$ and $1 \mathrm{M} \mathrm{NaOH}$ solution. The area of the working electrode for growth of nanowires was $0.608 \mathrm{~cm}^{2}\left(=0.25 \pi(0.88 \mathrm{~cm})^{2}\right)$ and the area of the graphite counter electrode was $14.7 \mathrm{~cm}^{2}$ $(=4.2 \mathrm{~cm} \times 3.5 \mathrm{~cm})$.

The $\mathrm{ZnCo}$ alloy nanowires were characterized by X-ray diffraction (XRD, Y-2000) with $\mathrm{CuK} \alpha$ radiations. The morphology of deposited nanowires was obtained by Scanning Electron Microscope (SEM, JEOL JSM-6700F) and transmission electron microscope (TEM, JEOL JEM2010). The AAO templates were partly dissolved in $5 \mathrm{wt} \%$ $\mathrm{NaOH}$ solution for SEM observations, and for TEM fully dissolved in the same solution, and then carefully rinsed with deionized water for several times.

\section{Results}

Figure 1 shows a typical SEM image of $\mathrm{ZnCo}$ alloy nanowires. The top view Figure 1 (a) of $\mathrm{ZnCO}$ alloy nanowires illustrates the flat heads of the electrodeposited nanowires and confirms that the diameter of the $\mathrm{ZnCo}$ alloy nanowires $(\sim 50 \mathrm{~nm})$ is the same as that of the nanopores of AAO template. Figure 1 (b) shows the cross-sectional view of electrodeposited nanowires indicating that the nanopores of the AAO template were fully filled with $\mathrm{Zn}-\mathrm{Co}$ atoms during electrodeposition. 


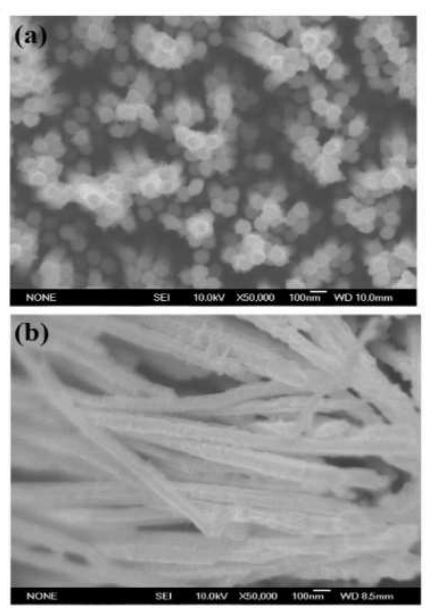

Figure 1. SEM images of ZnCo nanowires deposited at $-1.6 \mathrm{~V}$ and $\mathrm{Co}_{95 \mathrm{~g} / \mathrm{l}}$ $\mathrm{Zn}_{5 \mathrm{~g} / \text {; ( }}$ (a) top view and (b) cross-sectional view of $\mathrm{ZnCo}$ alloy nanowires.

The structural analysis of ZnCo alloy nanowires at various concentrations was studied by XRD and is shown in Figure 2. XRD results of $\mathrm{ZnCo}$ alloy nanowires electrodeposited at $1.6 \mathrm{~V}, \mathrm{pH} 3$ from electrolyte containing various concentrations $\quad\left(\mathrm{Co}_{95 \mathrm{~g} / 1} \mathrm{Zn}_{5 \mathrm{~g} / 1}\right), \quad\left(\mathrm{Co}_{90 \mathrm{~g} / 1} \mathrm{Zn}_{10 \mathrm{~g} / 1}\right), \quad$ and $\left(\mathrm{Co}_{85 \mathrm{~g} / 1} \mathrm{Zn}_{15 \mathrm{~g} / 1}\right)$ elaborate the role of $\mathrm{Zn}^{+2}$ ions in $\mathrm{ZnCo}$ alloy nanowires during electrodeposition process.

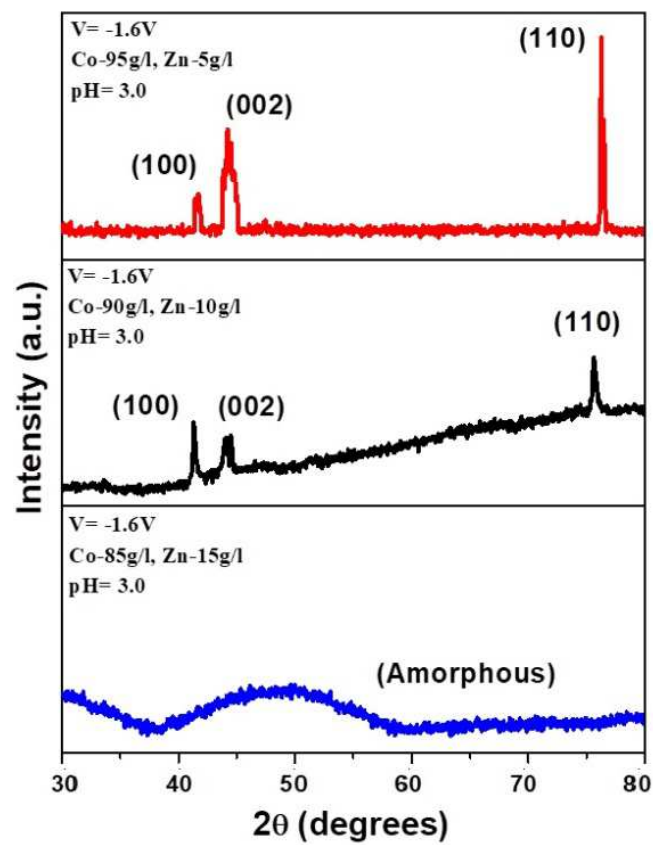

Figure 2. XRD pattern of $\mathrm{ZnCo}$ alloy nanowires at $\mathrm{pH} 3$ and voltage $-1.6 \mathrm{~V}$. (a) $\mathrm{Co}_{95 \mathrm{~g} / \mathrm{l}} \mathrm{Zn}_{5 \mathrm{~g} / \mathrm{l}}$, (b) $\mathrm{Co}_{90 \mathrm{~g} / \mathrm{l}} \mathrm{Zn_{10 \textrm {g } / l } )}$ and (c) $\mathrm{Co}_{85 \mathrm{~g} / \mathrm{Z}} \mathrm{Zn}_{15 \mathrm{~g} / \text { l. }}$

The XRD data were collected from the top side of nanowires. In Figure $2(\mathrm{a})$, three peaks at $2 \theta=41.6^{\circ} ; \mathrm{d}=2.16$, $2 \theta=44.6^{\circ} ; \mathrm{d}=2.02$, and $2 \theta=75.8^{\circ} ; \mathrm{d}=1.25$ were seen and can be attributed to (100), (002) and (110) planes of hep Co nanowires (Standard card \# 89-7094) for the $\mathrm{ZnCo}$ nanowires deposited in $\mathrm{Co}_{95 \mathrm{~g} / \mathrm{Z}} \mathrm{Zn}_{5 \mathrm{~g} / \mathrm{l}}$ solution at $-1.6 \mathrm{~V}$ with $\mathrm{pH} 3$ at room temperature. By increasing the concentration to $\mathrm{Co}_{90 \mathrm{~g} / 1} \mathrm{Zn}_{10 \mathrm{~g} / 1}$ the structure of the $\mathrm{ZnCo}$ nanowires remains same, however the intensity of the peaks decreases by increasing the $\mathrm{Zn}^{+2}$ ions concentration in the electrolyte as can be seen in Figure 2 (b). Further increase in concentration to $\mathrm{Co}_{85 \mathrm{~g} / 1} \mathrm{Zn}_{15 \mathrm{~g} / 1}$ the structure of $\mathrm{ZnCo}$ alloy nanowires there is no characteristic peak revealing the amorphous phase of $\mathrm{ZnCo}$ nanowires (see Figure 2 (c)). These results are in good agreement with the previous results [15], however the reason for this change phase is not well understood.

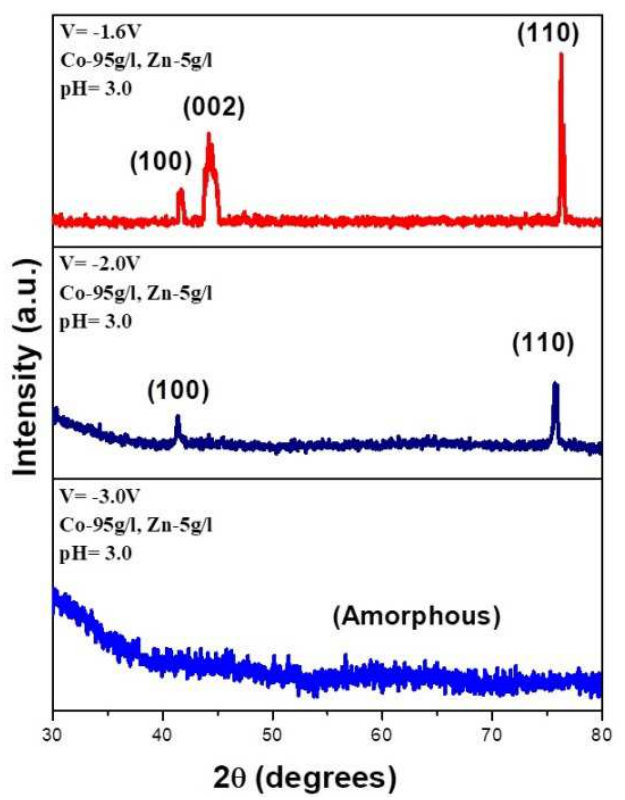

Figure 3. XRD pattern of $\mathrm{ZnCo}$ alloy nanowires at $\mathrm{pH}$ 3, concentration $\mathrm{Co}_{95 \mathrm{~g} / \mathrm{Z}} \mathrm{Zn}_{5 \mathrm{~g} / \text {, }}$ and at room temperature; (a)-1.6V (b) $-2.0 \mathrm{~V}$ and (c)-3.0 V.

Figure 3 shows the XRD pattern of $\mathrm{ZnCo}$ alloy nanowires at various potentials $(-1.6 \mathrm{~V},-2.0 \mathrm{~V}$ and $-3.0 \mathrm{~V})$ with $\mathrm{pH} 3$ at room temperature from the bath of concentration $\mathrm{Co}_{95 \mathrm{~g} / 1} \mathrm{Zn}_{5 \mathrm{~g} / \mathrm{l}}$. The effect of potential on the structure of $\mathrm{ZnCo}$ alloy nanowires is similar to that of concentration effect. Figure 3 (a) shows three peaks at $2 \theta=41.6^{\circ} ; \mathrm{d}=2.16,2 \theta=44.6^{\circ}$; $\mathrm{d}=2.02$, and $2 \theta=75.8^{\circ} ; \mathrm{d}=1.25$ and can be attributed to (100), (002) and (110) planes of hcp Co nanowires (Standard card \# 89-7094) for the $\mathrm{ZnCo}$ nanowires deposited at $-1.6 \mathrm{~V}$ form $\mathrm{Co}_{95 \mathrm{~g} / 1} \mathrm{Zn}_{5 \mathrm{~g} / 1}$ solution with $\mathrm{pH}$ 3. By increasing the potential to $-2.0 \mathrm{~V}$ (Figure 3 (b), the intensity of the peaks decreases and only two peaks (100) and (110) planes were seen. Further increase in potential to $-3.0 \mathrm{~V}$ as can be seen in Figure 3 (c) the structure changes to amorphous.

\section{Discussion}

Experimental results of $\mathrm{ZnCo}$ alloy nanowires clarify that change in concentration and potential can transform the crystal structure of electrodeposited $\mathrm{ZnCo}$ from crystalline to amorphous. Higher concentration of $\mathrm{Zn}^{+2}$ ions can lead to amorphous structure of $\mathrm{ZnCo}$ alloy nanowires, also the higher potential has similar effect to that of concentration effect. Electrodeposition technique is based on reaction, diffusion, adsorption and collaboration with the Al-substrate, concentration ratio of deposited alloy, applied voltage and electrolyte concentration. Thus the structure of the deposited alloy nanowires is associated to the deposition parameters 
and growth modes [26, 27]. The deposited phase of $\mathrm{ZnCo}$ alloy and its growth rate have a main role in defining the complete deposition cause, properties and morphology of deposited nanowires [28]. This can be explained as follows.

Effect of variable concentration and voltage on electrodeposited $\mathrm{ZnCo}$ alloy nanowires is explained according to electron tunneling. Electron tunneling elaborates the electronic mechanism occurring adjacent to the metal surface [29].

Electrodeposited metals include the reduction of metal ions from aqueous electrolytes. A simplified atomistic representation of reduction reaction is [30].

$$
\mathrm{M}_{\text {solution }}^{\mathrm{z}+}+\mathrm{ze} \rightarrow \mathrm{M}_{\text {lattice }}
$$

In this reaction, $z$ electrons (e) are supplied from an external power supply. The metal ions in an electrolytic solution (aqueous $M_{\text {solution }}^{Z+}$ ) are not bare metal ions but hydrated metal ions. The growth of electrodeposited metal nanowires accomplished in four main steps at atomic level.

In the electrodeposition of metal, a metal ion is transferred from solution into the ionic metal lattice, meanwhile electrons are provided from the external electron source (power supply) to the electron gas of the metal [31]. This electrodeposition process can be pictured as the following four steps in the atomic-level scale. First, hydrated metal ions in an aqueous solution diffuse to a metal surface and are adsorbed on this surface. (In hydrated metal ions, the water molecules of hydration are electrostatically attached to the metal ions.) Second, when an adsorbed hydrated metal ion captures electrons from the surface by quantum-mechanical tunneling, the metal ion becomes a neutral metal atom. The electrostatic attractive interaction between the neutral metal atom and water molecules is zero and the water molecules of hydration are displaced. Third, the neutral metal atom adsorbed on the surface. Fourth, an adsorbed metal atom diffuses to a surface site (such as kink site) where it incorporates into the ionic metal lattice, leading to the growth of nanowires. The growth arises from valence electron tunneling from the metal surface to hydrated metal and hydrogen ions, leading metal nanowire growth and hydrogen evolution, respectively.

Previously, it was shown that the increase of $\mathrm{Zn}^{+2}$ ions in electrolyte can increase the deposition rate of $\mathrm{Zn}$ resulting of the amorphous phase of $\mathrm{ZnCo}$ alloy nanowires [15]. This study shows the similar effect as well as voltage effect. This can be explained based on workfunctions of materials; $\mathrm{Zn}$ $\left(\emptyset_{1}=4.3\right)$ and $\left.\emptyset_{2}=5.0\right)$. According to tunneling effect, wave function of valence electrons never drops to zero abruptly at surface of metals. The value of wave function increase above the surface with an exponentially increase of decaying tail [32]. Valence electron tunnel to unoccupied orbit of hydrated metal ion. The primary step to calculate tunneling probability, is measured using wave functions. Tunneling probability increases by increasing the overlapping number among the orbital wavefunction and the valence electron wavefunction. The overlapping based on extra time required to decaying tail and the gap of hydrated metal ions from surface. According to wavefunction equation, electrons above the surface loose energy exponentially [33]. The workfunction of metal can have a significant effect on the barrier width that electrons tunnel through. Figure 4 (a) shows that under the same applied potential the barrier width (d) for the metal with smaller workfunction becomes thinner and electron tunneling occurs more easily. The greater the value of workfunction smaller will be the tunneling distance and faster will be the growth of that material. Since $\mathrm{Zn}$ has smaller workfunction and can grow faster which changes the crystallinity of $\mathrm{ZnCo}$ alloy nanowires. Therefore, by increasing the concentration of $\mathrm{Zn}^{+2}$ in electrolyte from $5 \mathrm{~g} / 1$ to $15 \mathrm{~g} / 1$ results in the amorphous phase of $\mathrm{ZnCo}$ alloy nanowires. The probability of electron tunneling is associated to the work function of electrodeposited material. Experimental results illustrates that the work function of metal ( $\mathrm{Zn}, 4.33$ and $\mathrm{Co}, 5.0)$ alloy have an important effect in electrodepositing metal growth on crystal surface $[25,34]$ as can be seen schematically in Figure 4 (a).
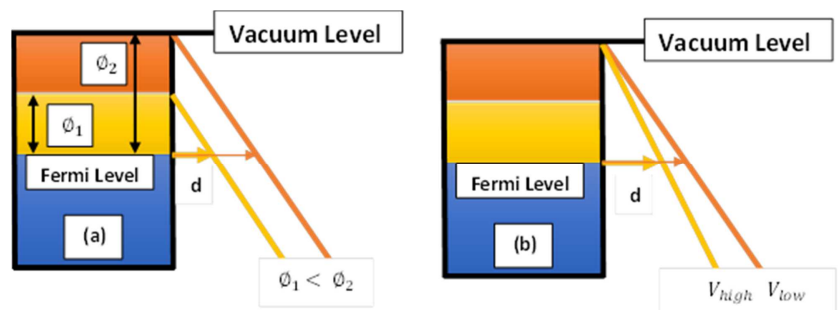

Figure 4. Schematic representation of electron tunneling: (a) electron tunneling occurs at fermi level and barrier thickness (d) decreases with difference of workfunction value, (b) the increase in potential makes the tunneling barrier thinner.

Therefore, under the same applied potential, there is a probability that $\mathrm{Zn}$ deposited faster than the Co leading the increase content in ZnCo alloy nanowires, [15] which further leads the amorphous phase.

The free electron gas model revealed, the metal can be taken as a potential box, filled with electrons to the Fermi level, which are below the vacuum level having path difference covered by several electronvolts. Work function is defined as the path from Fermi to vacuum level. High electric field is induced in between vacuum and fermi level named as double layer.

$$
E=-V / d
$$

In this equation $\mathrm{V}$ is applied voltage supply to metal electrodes, considered as voltage of electrolytic solution is zero and $\mathrm{d}$ is the total path in double layer. The variable field strength alternates the potential energy $E$ of the electrons outside of the metal basically calculated as $E=e \cdot \varepsilon \cdot x$ (where $\mathrm{x}$ is the distance from metal distance). So, electron tunneling is based on the strength of applied electric field [25]. From above equation, we can see that increasing the applied potential will make the electric field strength stronger. This leads to the more rapid change of the potential of electrons outside the metal, as shown in Figure 4 (b). In 
this case, the slope of electron potential with a higher electric field is steeper than that of with a small electric field. The barrier width that electrons tunnel through becomes thinner and electron tunneling occurs more easily. Therefore, at higher potential $-3.0 \mathrm{~V}, \mathrm{Zn}$ may be deposited more rapidly because of thinner barrier as the growth rate of $\mathrm{Zn}$ is higher than $\mathrm{Co}$ at same concentration. Consequently, changes the crystalline structure of $\mathrm{ZnCo}$ alloy nanowires to amorphous phase.

\section{Conclusion}

We have studied the structure of ZnCo alloy nanowires by varying concentration and potential. It was found that increase concentration $\mathrm{Zn}^{+2}$ results in the amorphous phase of $\mathrm{ZnCo}$ alloy nanowires. This can be explained by electron tunneling theory and workfunction effect. The smaller workfunction $(\mathrm{Zn}=4.3)$ increases the growth rate of $\mathrm{Zn}$ content, which leads to the amorphous phase. Furthermore, at same concentration the tunneling distance becomes steeper which increases the growth rate of $\mathrm{Zn}$, ultimately changes the structure of $\mathrm{ZnCo}$ alloy nanowires. The electron tunneling theory is illustrated (not drawn on scale) in very simple and easy way to understand.

\section{Acknowledgements}

The authors are grateful to the financial support by Natural Science Foundation of China, (Grant No. U1532268) and the Wuhan Science and Technology Program (Grant No. 2019010701011382).

\section{References}

[1] Y. Huang, X. Duan, Y. Cui, L. J. Lauhon, K.-H. Kim, and C. M. Lieber, "Logic Gates and Computation from Assembled Nanowire Building Blocks," Science, vol. 294, pp. 1313-1317, November 9, 20012001.

[2] X. Duan, Y. Huang, and C. M. Lieber, "Nonvolatile Memory and Programmable Logic from Molecule-Gated Nanowires," Nano Lett, vol. 2, pp. 487-490, 2002/05/01 2002.

[3] M. S. Gudiksen, L. J. Lauhon, J. Wang, D. C. Smith, and C. M. Lieber, "Growth of nanowire superlattice structures for nanoscale photonics and electronics," Nature, vol. 415, pp. 617-620, 2002.

[4] D. Xiangfeng, H. Yu, C. Yi, W. Jianfang, and M. L. Charles, "Indium phosphide nanowires as building blocks for nanoscale electronic and optoelectronic devices," Nature, vol. 409, pp. 66-69, 2001.

[5] M. Ding, H. Kim, and A. I. Akinwande, "Observation of valence band electron emission from n-type silicon field emitter arrays," Applied Physics Letters, vol. 75, pp. 823-825, 08/09/ 1999.

[6] F. C. K. Au, K. W. Wong, Y. H. Tang, Y. F. Zhang, I. Bello, and S. T. Lee, "Electron field emission from silicon nanowires," Applied Physics Letters, vol. 75, pp. 1700-1702, 09/20/ 1999.
[7] J. Zhao, C. Buia, J. Han, and J. P. Lu, "Quantum transport properties of ultrathin silver nanowires," Nanotechnology, vol. 14, pp. 501-504, 2003.

[8] W. U. Huynh, J. J. Dittmer, and A. P. Alivisatos, "Hybrid nanorod-polymer solar cells," Science, vol. 295, pp. 2425-7, Mar 292002.

[9] Y. Wu, R. Fan, and P. Yang, "Block-by-Block Growth of Single-Crystalline $\mathrm{Si} / \mathrm{SiGe}$ Superlattice Nanowires," Nano Lett, vol. 2, pp. 83-86, 2002.

[10] S. Nicewarner Pena, R, et al., in Encyclopedia of nanoscience and nanotechnology vol. 6, ed. Valencia, California, USA: American Scientific Publishers, 2004, p. 215.

[11] G. Chen, M. S. Dresselhaus, G. Dresselhaus, J. P. Fleurial, and T. Caillat, "Recent developments in thermoelectric materials," International Materials Reviews, vol. 48, pp. 45-66, // 2003.

[12] T. Thurn-Albrecht, J. Schotter, G. A. Kästle, N. Emley, T. Shibauchi, L. Krusin-Elbaum, et al., "Ultrahigh-Density Nanowire Arrays Grown in Self-Assembled Diblock Copolymer Templates," Science, vol. 290, pp. 2126-2129, December 15, 20002000.

[13] K. Nielsch, R. B. Wehrspohn, S. F. Fischer, H. Kronmüller, J. Barthel, J. Kirschner, et al., "Magnetic Properties of 100 NMPeriod Nickel Nanowire Arrays Obtained from Ordered Porous-Alumina Templates," MRS Online Proceedings Library, vol. 636, pp. null-null, 2000.

[14] Y. Zhu, J. Zhou, and H. Wang, "Influence of applied voltage on the characterization of direct current electrodeposited CoZn alloy nanowires," Materials Letters, vol. 210, pp. 191-193, 2018.

[15] M. Koohbor, S. Soltanian, M. Najafi, and P. Servati, "Fabrication of CoZn alloy nanowire arrays: Significant improvement in magnetic properties by annealing process," Materials Chemistry and Physics, vol. 131, pp. 728-734, 2012.

[16] M. H. Gharahcheshmeh and M. H. Sohi, "Pulse electrodeposition of $\mathrm{Zn}-\mathrm{Co}$ alloy coatings obtained from an alkaline bath," Materials Chemistry and Physics, vol. 134, pp. 1146-1152, 2012.

[17] Y. Xu, J. Fu, D. Gao, and D. Xue, "The fabrication and characteristic properties of amorphous Co1- xZnx alloy nanowire arrays," Journal of Alloys and Compounds, vol. 495, pp. 450-452, 2010.

[18] I. H. Karahan, O. Karabulut, and U. Alver, "A study on electrodeposited Zn-Co alloys," Physica Scripta, vol. 79, p. 055801, 2009.

[19] M. Almasi-Kashi, A. Ramazani, and M. Amiri-Dooreh, "FORC investigation of as-deposited and annealed CoZn alloy nanowires," Physica B: Condensed Matter, vol. 452, pp. 124130, 2014.

[20] M. A. Kashi, A. Ramazani, and Z. Fallah, "Magnetic properties improvement through off time between pulses and annealing in pulse electrodeposited CoZn nanowires," Journal of Alloys and Compounds, vol. 509, pp. 8845-8849, 2011.

[21] S. K. Lower, Electrochemistry. Textbook: Simon Fraser University. 
[22] M. Tan and X. Q. Chen, "Growth Mechanism of Single Crystal Nanowires of fcc Metals (Ag, Cu, Ni) and hcp Metal (Co) Electrodeposited," Journal of The Electrochemical Society, vol. 159, p. K15, 2012.

[23] T Mehmood, BS Khan, A Mukhtar, M Tan "Influence of bath temperature and $\mathrm{pH}$ on the structure of electrodeposited cobalt nanowires" International Journal of Materials Research vol.106, pp. 957, 2015.

[24] T. Mehmood, B. Shahzad Khan, A. Mukhtar, X. Chen, P. Yi, and M. Tan, "Mechanism for formation of fcc-cobalt nanowires in electrodeposition at ambient temperature," Materials Letters, vol. 130, pp. 256-258, 9/1/2014.

[25] B. Shahzad Khan, T. Mehmood, A. Mukhtar, and M. Tan, "Effect of workfunction on the growth of electrodeposited $\mathrm{Cu}$, $\mathrm{Ni}$ and Co nanowires," Materials Letters, vol. 137, pp. 13-16, $12 / 15 / 2014$.

[26] A. Krause, M. Uhlemann, A. Gebert, and L. Schultz, "A study of nucleation, growth, texture and phase formation of electrodeposited cobalt layers and the influence of magnetic fields," Thin Solid Films, vol. 515, pp. 1694-1700, 2006.

[27] A. Vicenzo and P. L. Cavallotti, "Growth modes of electrodeposited cobalt," Electrochimica Acta, vol. 49, pp. 4079-4089, 2004.
[28] P. L. Cavallotti, A. Vicenzo, M. Bestetti, and S. Franz, "Microelectrodeposition of cobalt and cobalt alloys for magnetic layers," Surface and Coatings Technology, vol. 169, pp. 76-80, 2003.

[29] B. S. Khan, T. Mehmood, A. Mukhtar, and M. Tan, "Effect of workfunction on the growth of electrodeposited $\mathrm{Cu}, \mathrm{Ni}$ and $\mathrm{Co}$ nanowires," Materials Letters, vol. 137, pp. 13-16, 2014.

[30] M. Paunovic and M. Schlesinger, "Fundamentals of electrochemical deposition," New York, 1998.

[31] Milan Paunovic and M. Schlesinger, "Fundamentals of Electrochemical Deposition," Fundamentals of Electrochemical Deposition, New York: Wiley., 1998.

[32] L. E. C. v. d. Leemput and H. v. Kempen, "Scanning tunnelling microscopy," Reports on Progress in Physics, vol. 55, p. 1165, 1992.

[33] S. J. Garrett, Introduction to Surface Analysis. the Michigan State University, Chemistry Department, East Lansing, 2001.

[34] N. Lang and W. Kohn, "Theory of metal surfaces: work function," Physical Review B, vol. 3, p. 1215, 1971. 